\title{
Average bit error probability for the $\lambda$-MRC detector under Rayleigh fading
}

\author{
Mitchell Omar Calderon Inga and Gustavo Fraidenraich*
}

\begin{abstract}
In this paper, an exact expression for the average bit error probability was obtained for the $\lambda$-MRC detector, proposed in Sendonaris et al. (IEEE Trans Commun 51: 1927-1938, IEEE Trans. Commun 51: 1939-1948), under Rayleigh fading channel. In addition, a very accurate approximation was obtained to calculate the average bit error probability for any power allocation scheme. Our expressions allow to investigate the possible gains and situations where cooperation can be beneficial.
\end{abstract}

Keywords: User cooperation, Virtual MIMO, Bit error probability, Rayleigh fading

\section{Introduction}

Diversity techniques have been widely accepted as one of effective ways of combat multipath fading in wireless communications [1], in particular spatial diversity is specially effective at mitigating these multipath situation. However, in many wireless applications, the use of multiple antennas is not practical due to size and cost limitations of the terminals. One possible way to have diversity without increasing the number of antennas is through the use of cooperative diversity.

Cooperative diversity has root in classical information theory work on relay channels [2], [3]. Cooperative networks achieve diversity gain by allowing the users to cooperate, and thus, each wireless user is assumed to transmit data as well as act as a cooperative agent for another user [4], [5]. The first implementation strategy for cooperation was introduced in [1], [6], where the achievable rate region, outage probability, and coverage area were analyzed.

In this pioneering work, assuming a suboptimal receiver called $\lambda$-MRC, the bit error probability was computed assuming a fixed channel. This kind of receiver combines the signal from the first period of transmission with the signal transmitted jointly by the both users in the second period of transmission. The variable $\lambda \in[0,1]$ establishes the degree of confidence in the bits estimated by the partner. For situations where the interuser channel presents favorable conditions, the variable

\footnotetext{
* Correspondence: gf@decom.fee.unicamp.br

Department of Communications, University of Campinas, Campinas, Brazil
}

$\lambda$ should be close to unity; on the other hand, for very severe channels conditions, the parameter $\lambda$ should tend to zero. Unfortunately, the bit error probability was computed only for a fixed channel and remained open for the situation where all the fading coefficients are Rayleigh distributed.

In this paper, an exact and approximate expression is computed for the average bit error probability assuming a Rayleigh fading for the inter-user channel and for the direct channel between users and base station (BS).

\section{System Model}

This section summarizes the system model that was employed in [1], [6].

\section{A. System Model}

The channel model used in [6] can be mathematically expressed as

$$
\begin{aligned}
& Y_{0}(t)=K_{10} X_{1}(t)+K_{20} X_{2}(t)+Z_{0}(t) \\
& Y_{1}(t)=K_{21} X_{2}(t)+Z_{1}(t) \\
& Y_{2}(t)=K_{12} X_{1}(t)+Z_{2}(t)
\end{aligned}
$$

where $Y_{0}(t), Y_{1}(t)$, and $Y_{2}(t)$ are the baseband models of the received signal at the BS, user 1 , and user 2 , respectively, during one symbol period. Also, $X_{i}(t)$ is the signal transmitted by user $i$ under power constraint $P_{i}$, for $i=1,2$, and $Z_{i}(t)$ are white zero-mean Gaussian noise random processes with spectral height $\mathcal{N}_{i} / 2$ for $i$ 
$=0,1,2$, and the fading coefficients $K_{i j}$ are Rayleigh distributed with $E\left[K_{i j}^{2}\right]=2 \alpha_{i j}^{2}$. We also assume that the BS can track perfectly the variations in $K_{10}$ and $K_{20}$, user 1 can track $K_{21}$ and user 2 can track $K_{12}$.

The system proposed in [6] is based on a conventional code division multiple access (CDMA) system and divides the transmission into two parts: the first without cooperation and the second with cooperation. For a given coherence time of $L$ symbols and cooperation time of $2 L_{c}$ symbols, the transmitted signals can be expressed as shown in (5), where $L_{n}=L-2 L_{c}, b_{j}^{(i)}$ is user $j$ 's $i$ th bit, $\hat{b}_{j}^{(i)}$ is the partner's estimate of user $j$ 's $i$ th bit, and $c_{j}(t)$ is user $j$ 's spreading code. The parameters $a_{i j}$ represent the power allocation scheme, and they must maintain an average power constraint that can be expressed as

$$
\begin{aligned}
& \frac{1}{L}\left(L_{n} a_{11}^{2}+L_{c}\left(a_{12}^{2}+a_{13}^{2}+a_{14}^{2}\right)\right)=P_{1} \\
& \frac{1}{L}\left(L_{n} a_{21}^{2}+L_{c}\left(a_{22}^{2}+a_{13}^{2}+a_{14}^{2}\right)\right)=P_{2} \\
& X_{1}(t)= \begin{cases}a_{11} b_{1}^{(i)} c_{1}(t), & i=1,2, \ldots, L_{n} \\
a_{12} b_{1}^{\left(L_{n}+1+1\right) / 2} c_{1}(t), & i=L_{n}+1, L_{n}+3, \ldots, L-1 \\
a_{13} b^{\left(L_{n}+i\right) / 2} c_{1}(t)+a_{14} \hat{b}_{2}^{\left(L_{n+1}\right) / 2} c_{2}(t), & i=L_{n}+2, L_{n}+4, \ldots, L\end{cases} \\
& X_{2}(t)= \begin{cases}a_{21} b_{2}^{(i)} c_{2}(t) / 2 & i=1,2, \ldots, L_{n} \\
a_{22} b_{1}^{\left(n_{n}+1+i\right) / 2} c_{2}(t), & i=L_{n}+1, L_{n}+3, \ldots, L-1 \\
a_{23} \hat{b}_{1}^{\left(L_{n}+i\right) / 2} c_{1}(t)+a_{24} b_{2}^{\left(L_{n+1}\right) / 2} c_{2}(t), & i=L_{n}+2, L_{n}+4, \ldots, L\end{cases}
\end{aligned}
$$

In the first $L_{n}=L-2 L_{c}$ symbol periods, each user transmits its own bits to the BS. The remaining $2 L_{c}$ periods are dedicated to cooperation: odd periods for transmitting its bits to both the partner and the BS; even periods for transmitting a linear combination of its own bit and the partner's bit estimate.

\section{B. Error Calculations}

1) Error Rate for Cooperative Periods: During the $2 L_{\mathrm{c}}$ cooperative periods, we have a distinction between "odd" and "even" periods. During the "odds" periods, each user sends only their own bit, which is received and detected by the partner as well as by the BS.

The partner's hard estimate of $b_{1}$ is given by $\hat{b}_{1}=\operatorname{sign}\left(\left(1 / N_{c}\right) c_{1}^{T} Y_{2}\right)$, resulting in a probability of bit error equals to

$$
P_{e_{12}}=Q\left(K_{12} a_{12} \frac{\sqrt{N_{c}}}{\sigma_{2}}\right)
$$

where $Q(\cdot)$ is the Gaussian error integral, $N_{c}$ is the CDMA spreading gain, $\sigma_{2}^{2}=\mathcal{N}_{2} /\left(2 T_{c}\right), T_{c}$ is the chip period, and $\mathcal{N}_{2} / 2$ is the spectral height of $Z_{2}(t)$.
The BS forms a soft decision statistic by calculating

$$
y_{\text {odd }}=\frac{1}{N_{c}} c_{1}^{T} Y_{0}^{\text {odd }}
$$

where $Y_{0}^{\text {odd }}=K_{10} X_{1}+K_{20} X_{2}+Z_{0}^{\text {odd }}$.

During the "even" periods, each user send a cooperative signal to BS according to $Y_{0}^{\text {even }}=K_{10} X_{1}+K_{20} X_{2}+Z_{0}^{\text {even }}$, and the BS extracts a soft decision statistic by calculating

$$
y_{\text {even }}=\frac{1}{N_{c}} c_{1}^{T} Y_{0}^{\text {even }}
$$

The combined statistics at BS for user 1 is therefore given by

$$
\begin{aligned}
& y_{\text {odd }}=K_{10^{a} 12} b_{1}+n_{\text {odd }} \\
& y_{\text {even }}=K_{10^{a} 13} b_{1}+K_{20^{a} 23} \hat{b}_{1}+n_{\text {even }}
\end{aligned}
$$

where $n_{\text {odd }}$ and $n_{\text {even }}$ are statistically independent and both distributed according to a Gaussian distribution $\mathcal{N}\left(0, \sigma_{0}^{2} / N_{c}\right)$.

The optimal detector shown in [1] is rather complex and does not have a closed-form expression for the resulting bit error probability. Thus, they consider the following suboptimum detector

$$
\hat{b}_{1}=\operatorname{sign}\left(\left[\mathrm{K}_{10^{\mathrm{a}} 12} \lambda\left(\mathrm{K}_{10^{\mathrm{a}} 13}+\mathrm{K}_{20^{\mathrm{a}} 23}\right)\right] \mathrm{y}\right)
$$

where $\mathrm{y}=\left[y_{\text {odd }} y_{\text {even }}\right]^{T} \sqrt{N_{c}} / \sigma_{0}$ and $\lambda \in[0,1]$. They call this suboptimum detector as the $\lambda$-MRC. The probability of bit error for this detector is given by

$$
P_{e_{1}}=\left(1-P_{e_{12}}\right) Q\left(\frac{v_{\lambda}^{T} v_{1}}{\sqrt{v_{\lambda}^{T} v_{\lambda}}}\right)+P_{e_{12}} Q\left(\frac{v_{\lambda}^{T} v_{2}}{\sqrt{v_{\lambda}^{T} v_{\lambda}}}\right)
$$

where

$v_{\lambda}=\left[\begin{array}{lll}K_{10} a_{12} & \lambda\left(K_{10} a_{13}+K_{20} a_{23}\right)\end{array}\right]^{T}, \quad v_{1}=\left[\begin{array}{lll}K_{10} a_{12} & \lambda\left(K_{10} a_{13}+K_{20} a_{23}\right)\end{array}\right]^{T} \sqrt{N_{c}} / \sigma_{0}$ and $v_{2}=\left[\begin{array}{lll}K_{10} a_{12} & \left(K_{10} a_{13}-K_{20} a_{23}\right)\end{array}\right]^{T} \sqrt{N_{c}} / \sigma_{0}$.

\section{Rayleigh fading calculations}

The expression presented in (11) is only valid for a fixed (time-invariant) channel, that is, the fading coefficients $K_{i j}$ are fixed. The aim of this paper is to obtain an expression for the bit error probability when the fading coefficients vary according to a Rayleigh distribution.

\section{A. Bit Error Probability}

The bit error probability associated with the signal from user 1, at user 2, for a fixed gain is described in (6). Now assuming a nonstatic situation, the average bit error probability can be computed averaging (6) with respect to a Rayleigh distribution 


$$
\bar{P}_{e_{12}}=E\left[P_{e_{12}}\right]=\frac{1}{2}\left(1-\sqrt{\frac{\gamma_{12}}{2+\gamma_{12}}}\right)
$$

where $\gamma_{12}$ is the average signal-to-noise ratio, defined as

$$
\gamma_{12}=\frac{2 a_{12}^{2} \alpha_{12}^{2} N_{c}}{\sigma_{2}^{2}}
$$

From (11), we can define two random variables $U_{1}$ and $U_{2}$, respectively, as

$$
\begin{aligned}
& \frac{v_{\lambda}^{T} v_{1}}{\sqrt{v_{\lambda}^{T} v_{\lambda}}}=\sqrt{U_{1}}=\frac{\left(\left(K_{10} a_{12}\right)^{2}+\lambda\left(K_{10} a_{13}+K_{20} a_{23}\right)^{2}\right) \sqrt{N_{c}}}{\left(\left(K_{10} a_{12}\right)^{2}+\lambda^{2}\left(K_{10} a_{13}+K_{20} a_{23}\right)^{2}\right) \sigma_{0}}(14) \\
& \frac{v_{\lambda}^{T} v_{2}}{\sqrt{v_{\lambda}^{T} v_{\lambda}}}=\sqrt{U_{2}}=\frac{\left(\left(K_{10} a_{12}\right)^{2}+\lambda\left(\left(K_{10} a_{13}\right)^{2}-\left(K_{20} a_{23}\right)^{2}\right)\right) \sqrt{N_{c}}}{\left(\left(K_{10} a_{12}\right)^{2}+\lambda^{2}\left(K_{10} a_{13}+K_{20} a_{23}\right)^{2}\right) \sigma_{0}}
\end{aligned}
$$

since $K_{10}$ and $K_{20}$ are Rayleigh distributed, the support of (14) will be always greater than zero. On the other hand, since we have negative values in the numerator of (15), its support will be all the real line. Taking this into account, we can rewrite (11) as

$$
P_{e_{1}}=\left(1-P_{e_{12}}\right) Q\left(\sqrt{U_{1}}\right)+P_{e_{12}} Q\left(U_{2}\right)
$$

To obtain the error probability, we must average $P_{e_{1}}$, over the probability density function (PDF) of $U_{1}$ and $U_{2}$ [7]. Thus, we have to evaluate the integral

$$
\begin{array}{r}
P_{e_{f}}=\left(1-\bar{P}_{e_{12}}\right) \int_{0}^{\infty} Q\left(\sqrt{u_{1}}\right) f_{u_{1}}\left(u_{1}\right) d u_{1}+ \\
\bar{P}_{e_{12}} \int_{-\infty}^{\infty} Q\left(u_{2}\right) f_{u_{2}}\left(u_{2}\right) d u_{2}
\end{array}
$$

In order to calculate $P_{e f}$, we have to know the distribution of $U_{1}$ and $U_{2}$, thus to facilitate the calculations, we assume an equal power allocation situation, where $a_{12}=a_{13}=a_{23}=a$. With this assumption the random variables $U_{1}$ and $U_{2}$ will be simplified to

$$
\begin{gathered}
U_{1}=\frac{a^{2}\left(K_{10}^{2}+\lambda\left(K_{10}+K_{20}\right)^{2}\right) N_{c}}{\left(K_{10}^{2}+\lambda^{2}\left(K_{10}+K_{20}\right)^{2}\right) \sigma_{0}^{2}} \\
U_{2}=\frac{a\left(K_{10}^{2}+\lambda\left(K_{10}^{2}+K_{20}^{2}\right)\right) \sqrt{N_{c}}}{\left(\sqrt{K_{10}^{2}+\lambda^{2}\left(K_{10}+K_{20}\right)^{2}}\right) \sigma_{0}}
\end{gathered}
$$

Since $U_{1}$ depends on $K_{10}$ and $K_{20}$, it is possible to write the cumulative distribution function $(\mathrm{CDF})$ and the PDF of $U_{1}$, respectively, as

$$
\begin{aligned}
& F_{u_{1}}\left(u_{1}\right)=\iint_{k_{10}, k_{20} \in D_{u_{1}}} f_{u_{1}}\left(k_{10}, k_{20}\right) \mathrm{d} k_{10} \mathrm{~d} k 20 \\
& f_{u_{1}}\left(u_{1}\right)=\frac{\mathrm{d} F_{u_{1}}\left(u_{1}\right)}{d u_{1}}
\end{aligned}
$$

In this case, $D_{u_{1}}$ is the region of the $K_{10} \times K_{20}$ plane where

$$
\frac{a^{2}\left(k_{10}^{2}+\lambda\left(k_{10}+k_{20}\right)^{2}\right) N_{c}}{\left(k_{10}^{2}+\lambda^{2}\left(k_{10}+k_{20}\right)^{2}\right) \sigma_{0}^{2}} \leq u_{1}
$$

Note that this region is very similar to a rotated ellipse but not exactly an ellipse.

Since $K_{10}$ and $K_{20}$ are independent Rayleigh distribution with parameters $\alpha_{10}$ and $\alpha_{20}$, respectively, we have

$$
F_{u_{1}}\left(u_{1}\right)=\int_{k_{10}=0}^{a\left(u_{1}\right)} \int_{k_{20}=0}^{b\left(u_{1}\right)} f_{k_{10} k_{20}}\left(k_{10}, k_{20}\right) \mathrm{d} k_{20} \mathrm{~d} k_{10}
$$

where

$$
\begin{aligned}
& a\left(u_{1}\right)=\frac{1}{\lambda_{1}} \sqrt{\frac{u_{1} \lambda_{2}}{A_{1}}} \\
& b\left(u_{1}\right)=\frac{\sqrt{2 A_{1}\left(B_{1}-\left(2 A_{1} k_{10}^{2}-u_{1}\right) \lambda\right)}-2 A_{1} k_{10} \lambda^{2}}{2 A_{1} \lambda} \\
& A_{1}=\frac{a^{2} N_{c}}{\sigma_{0}^{2}} \\
& B_{1}=\lambda \sqrt{u_{1}\left(u_{1} \lambda^{2}-4 A_{1} k_{10}^{2}(\lambda-1)\right)}
\end{aligned}
$$

now it is possible to derive the PDF of $U_{1}$ easily as

$$
f_{u_{1}}\left(u_{1}\right)=\int_{k_{10}=0}^{a\left(u_{1}\right)} \frac{\partial b\left(u_{1}\right)}{\partial u_{1}} \frac{b\left(u_{1}\right)}{\alpha_{20}^{2}} e^{-\frac{b\left(u_{1}\right)^{2}}{2 \alpha_{20}^{2}}} \frac{k_{10}}{\alpha_{10}^{2}} e^{-\frac{k_{10}^{2}}{2 \alpha_{10}^{2}}} \mathrm{~d} k_{10}
$$

and unfortunately, it is not possible to evaluate (28) in a closed-form solution.

In order to validate the above formulation, Figure 1 shows the analytical and simulated PDF of $U_{1}$. Note the excellent agreement between them showing the correctness of our formulation.

Following similar rationale, we now find the CDF and PDF of $U_{2}$. Note that in this case, the region of integration, $D_{u_{2}}$, will be given by 


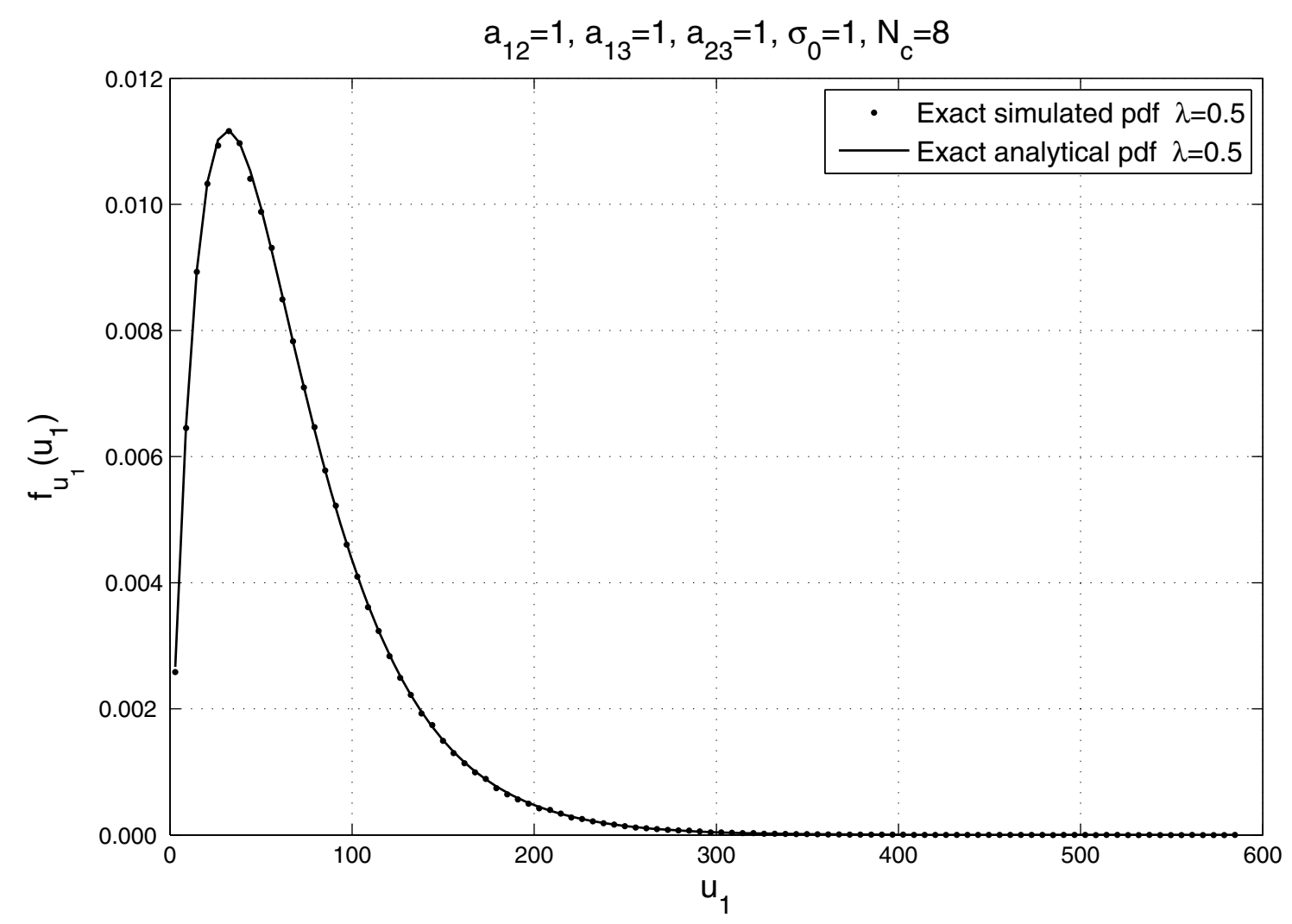

Figure 1 Comparison between analytical and simulated PDF for $U_{1}$

$$
\frac{a\left(k_{10}^{2}+\lambda\left(k_{10}^{2}-k_{20}^{2}\right)\right) \sqrt{N_{c}}}{\left(\sqrt{k_{10}^{2}+\lambda^{2}\left(k_{10}+k_{20}\right)^{2}}\right) \sigma_{0}} \leq u_{2}
$$

leading to the following CDF and PDF, respectively, as

$$
F_{u_{2}}\left(u_{2}\right)= \begin{cases}\int_{k_{20}=}^{\infty} \int_{\frac{\left|u_{2}\right|}{A_{2}}}^{\infty} \int_{k_{10}=0}^{\infty} f_{k_{10} k_{20}}\left(k_{10}, k_{20}\right) \mathrm{d} k_{10} \mathrm{~d} k_{20} & \text { if } u_{2}<0, \\ \int_{k_{20}=0}^{\infty} a \int_{k_{10}=0}^{a\left(u_{2}\right)} f_{k_{10} k_{20}}\left(k_{10}, k_{20}\right) \mathrm{d} k_{10} \mathrm{~d} k_{20} & \text { if } u_{2} \geq 0 .\end{cases}
$$

and

$$
f_{u_{2}}\left(u_{2}\right)= \begin{cases}\int^{\infty} \frac{\partial b\left(u_{2}\right)}{\partial u_{2}} f_{k_{10} k_{20}}\left(b\left(u_{2}\right), k_{20}\right) \mathrm{d} k_{20} \text { if } u_{2}<0, \\ \frac{\left|u_{2}\right|}{A_{2}} \\ \int_{0}^{\infty} \frac{\partial a\left(u_{2}\right)}{\partial u_{2}} f_{k_{10} k_{20}}\left(a\left(u_{2}\right), k_{20}\right) \mathrm{d} k_{20} & \text { if } u_{2} \geq 0 .\end{cases}
$$

where

$$
a\left(u_{2}\right)=\Re\left(\frac{1}{2 A_{2} \lambda_{1} \sqrt{3}}\left(\sqrt{R_{1}}+\sqrt{\frac{R_{2}}{2}}\right)\right)
$$

$$
b\left(u_{2}\right)=\Re\left(\frac{1}{2 A_{2} \lambda_{1} \sqrt{3}}\left(\sqrt{R_{1}}+\sqrt{\frac{R_{2}}{2}}\right)\right)
$$

$$
A_{2}=\frac{a \sqrt{N_{c}}}{\sigma_{0}}
$$

where $\Re(\cdot)$ denotes the real part of a number, and $R_{1}$ and $R_{2}$ are described in the Appendix.

In the same way as in the first case, (31) cannot be obtained in a closed-form solution. Figure 2 compares the analytical and simulated PDF of $U_{2}$ in order to validate our formulation.

Once that the PDFs of $U_{1}$ and $U_{2}$ were exactly computed, it is possible to obtain the average bit error probability by simply substituting (28) and (31) into (17). Figure 3 shows the simulation result of the bit error probability and the result of our theoretical expression given in (17), where we can observe that both curves are almost coincident. In this figure, $\mathrm{SNR}=\frac{P}{\sigma_{0}^{2}}$. According to Section II-B, we consider three symbols periods, each period with an average power of $P$. Also, for simplicity, 


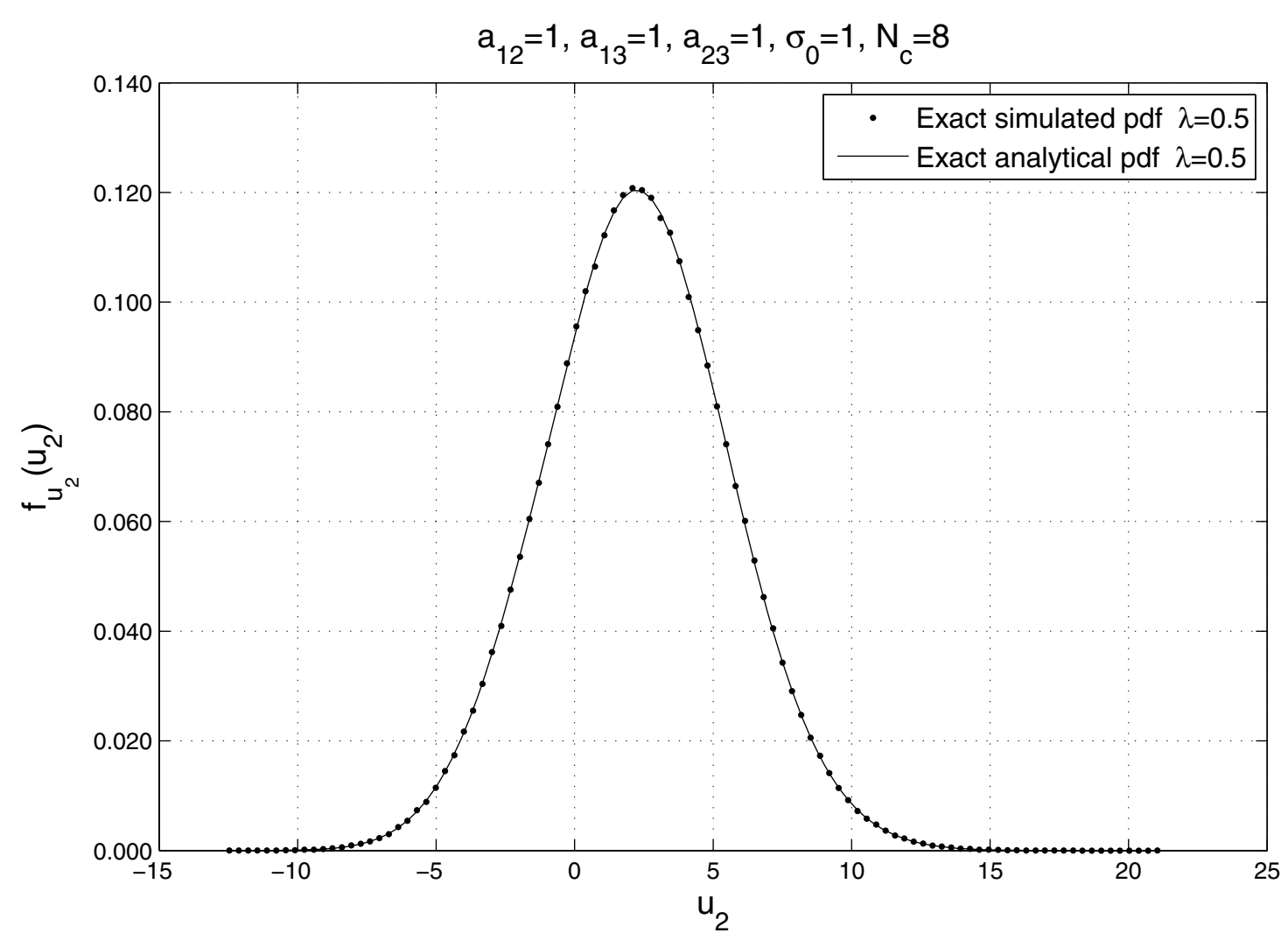

Figure 2 Comparison between the exact and simulated PDF for $\mathrm{U}_{2}$.

we consider that $E\left[K_{10}^{2}\right], E\left[K_{20}^{2}\right]$ and $E\left[K_{12}^{2}\right]$ are identical.

Although (17) presents the exact solution to the average bit error probability, in some cases, the complexity to compute this expression can be prohibitive. For this reason, we found a very accurate approximation for the bit error probability presented in the sequel.

\section{B. Approximate Bit error Probability}

The main problem in order to obtain a simpler expression for the bit error probability is to simplify the PDFs of $U_{1}$ and $U_{2}$ given, respectively, in (14) and (15). In order to obtain an approximation, the expressions (14) and (15) can be reduced when $\lambda=1, \sigma_{0}=1$ and $a_{12}=$ $a_{13}=a_{23}=1$. Therefore, the new random variables are given by

$$
\begin{aligned}
& U_{1}^{\prime}=N_{c}\left(K_{10}^{2}+\left(K_{10}+K_{20}\right)^{2}\right) \\
& U_{2}^{\prime}=\frac{\sqrt{N_{c}}\left(2 K_{10}^{2}-K_{20}^{2}\right)}{\sqrt{K_{10}^{2}+\left(K_{10}+K_{20}\right)^{2}}}
\end{aligned}
$$

Considering $D_{u^{\prime} 1}$ as the region of the plane $K_{10} \times K_{20}$ where $N_{c}\left(k_{10}^{2}+\left(k_{10}+k_{20}\right)^{2}\right) \leq u_{1}^{\prime}$, it can be seen that $D_{u^{\prime} 1}$ corresponds to the area of an ellipse whose center is in the origin $(0,0)$. Unfortunately, the evaluation of the integral (20) is rather complex for the domain $D_{u^{\prime} 1}$. For this reason, we consider a simplified version of $D_{u^{\prime} 1}$, as being the area of a circle expressed as $k_{10}^{2}+k_{20}^{2} \leq u_{1}^{\prime}$. This simplification can be applied since a circle corresponds to a particular case of the general ellipse. Hence

$$
F_{u_{1}^{\prime}}\left(u_{1}^{\prime}\right)=\int_{k_{20}=-\sqrt{u_{1}^{\prime}}}^{\sqrt{u_{1}^{\prime}}} \int_{k_{10}=-\sqrt{u^{\prime}{ }_{1}-k_{20}^{2}}}^{\sqrt{u_{1}^{\prime}-k_{20}^{2}}} f_{k_{10} k_{20}}\left(k_{10}, k_{20}\right) \mathrm{d} k_{10} \mathrm{~d} k_{20}
$$

This gives

$$
\begin{aligned}
& f_{u_{1}^{\prime}}\left(u_{1}^{\prime}\right)= \\
& \int_{k_{20}=-\sqrt{u_{1}^{\prime}}}^{\sqrt{u_{1}^{\prime}}} \frac{1}{2 \sqrt{u_{1}^{\prime}-k_{20}^{2}}}\left\{f_{k_{10} k_{20}}\left(\sqrt{u_{1}^{\prime}-k_{20}^{2}}, k_{20}\right)+\right. \\
& \left.f_{k_{10} k_{20}}\left(-\sqrt{u_{1}^{\prime}-k_{20}^{2}}, k_{20}\right)\right\} \mathrm{d} k_{20}
\end{aligned}
$$




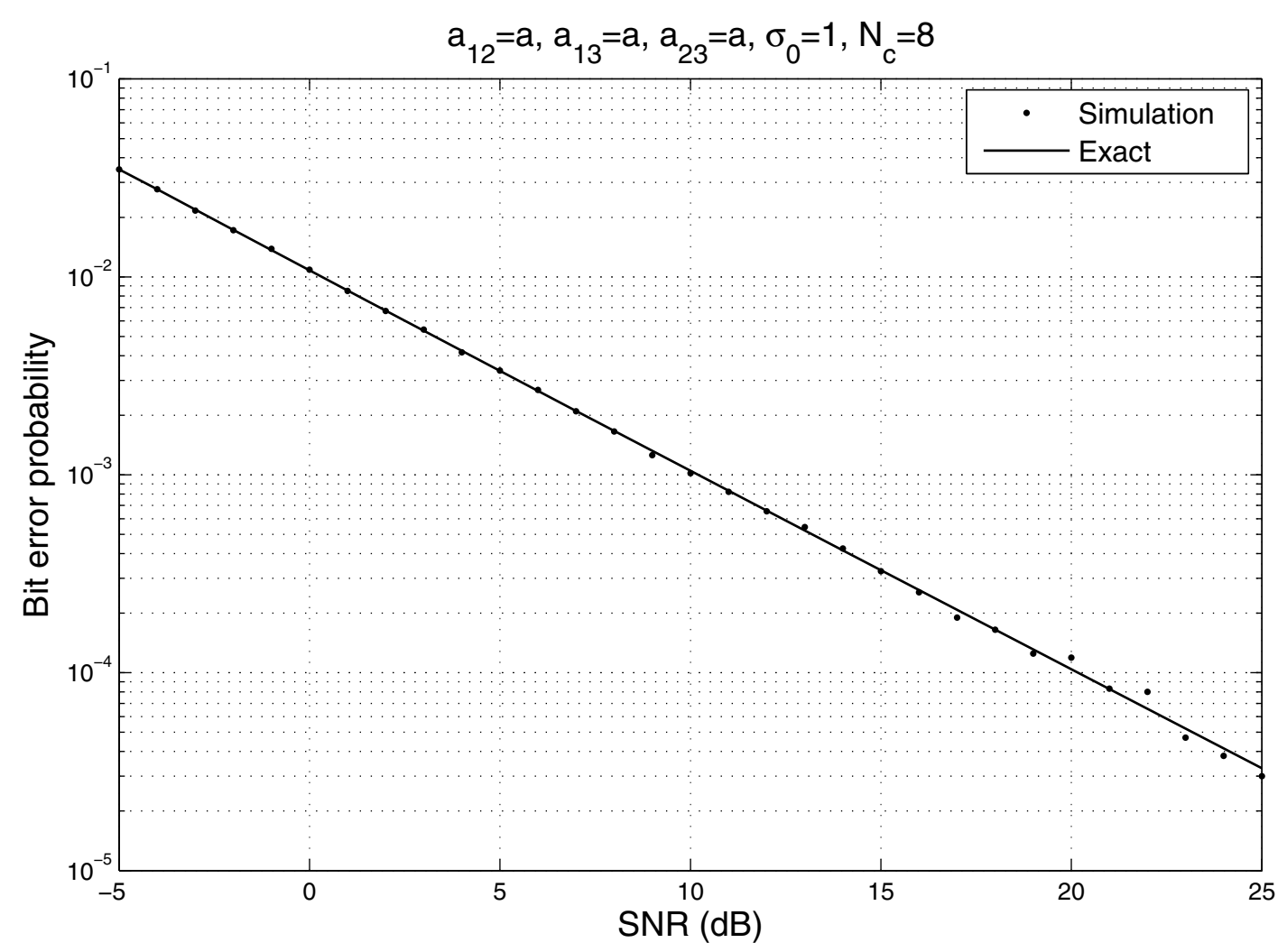

Figure 3 Comparison of the exact and simulated bit error probability adopting an equal power allocation scheme with $\lambda=0.5$

Since $K_{10}$ and $K_{20}$ are independent Rayleigh distributed with parameters $\alpha_{1}$ and $\alpha_{2}$, respectively, the PDF of $U_{1}^{\prime}$ given in (38) will result in a chi-square probability distribution with four degrees of freedom [8]. Therefore, our approximation of $U_{1}$ will be given by

$$
f_{u_{1}}\left(u_{1}\right) \approx \frac{4 u_{1}}{\gamma_{1}^{2}} e^{-2 u_{1} / \gamma_{1}}
$$

where $\gamma_{1}$ is the mean of $U_{1}$ given in (14)

$$
\gamma_{1}=E\left[U_{1}\right]
$$

Figure 4 shows the comparison between our approximate PDF given in (39) and the computer simulation for the PDF of $U_{1}$ given in (14) for two different values of $\lambda$ keeping the same values for $a_{12}=1, a_{13}=2$, and $a_{23}=3$. We observe that the curves are very close for both values of $\lambda$. Although only these two cases are presented here, many other cases were compared and the approximation still remains very good.

A similar rationale can be applied in order to find a good approximation for $U_{2}$. The region of the $K_{10} \times K_{20}$ plane where $U_{2}^{\prime} \leq u_{2}^{\prime}$ is similar to (29). Note that the range of $U_{2}{ }^{\prime}$ varies from $-\infty \leq u_{2}{ }^{\prime} \leq \infty$, discarding all the distributions with positive support. In order to observe the behavior of the PDF of $U_{2}{ }^{\prime}$, a large number of simulations were performed, and the Gaussian distribution proves to fit extremely well in all the cases. Therefore, assuming a Gaussian distribution, the following can be written

$$
P_{e_{f}} \approx \frac{1}{4}\left(1+\sqrt{\frac{\gamma_{12}}{2+\gamma_{12}}}\right)\left(1-\frac{\sqrt{\gamma_{1}}\left(\gamma_{1}+6\right)}{\left(\gamma_{1}+4\right)^{3 / 2}}\right)+\frac{1}{2}\left(1-\sqrt{\frac{\gamma_{12}}{2+\gamma_{12}}}\right) Q\left(\frac{\gamma_{2}}{\sqrt{1+v^{2}}}\right)
$$

$$
f_{u_{2}}\left(u_{2}\right) \approx \frac{1}{\sqrt{2 \pi v^{2}}} e^{-\frac{\left(u_{2}-\gamma_{2}\right)^{2}}{2 v^{2}}}
$$

where

$$
\begin{aligned}
& \gamma_{2}=E\left[U_{2}\right] \\
& v^{2}=\operatorname{var}\left(U_{2}\right)
\end{aligned}
$$

Figure 5 shows the comparison between the approximate PDF given in (42) and the computer simulation for the PDF of $U_{2}$ given in (15), for two different values of $\lambda$. Note that the approximation is less accurate for small values of $\lambda$, but this inaccuracy does not have a significant influence in the bit error probability. In all 


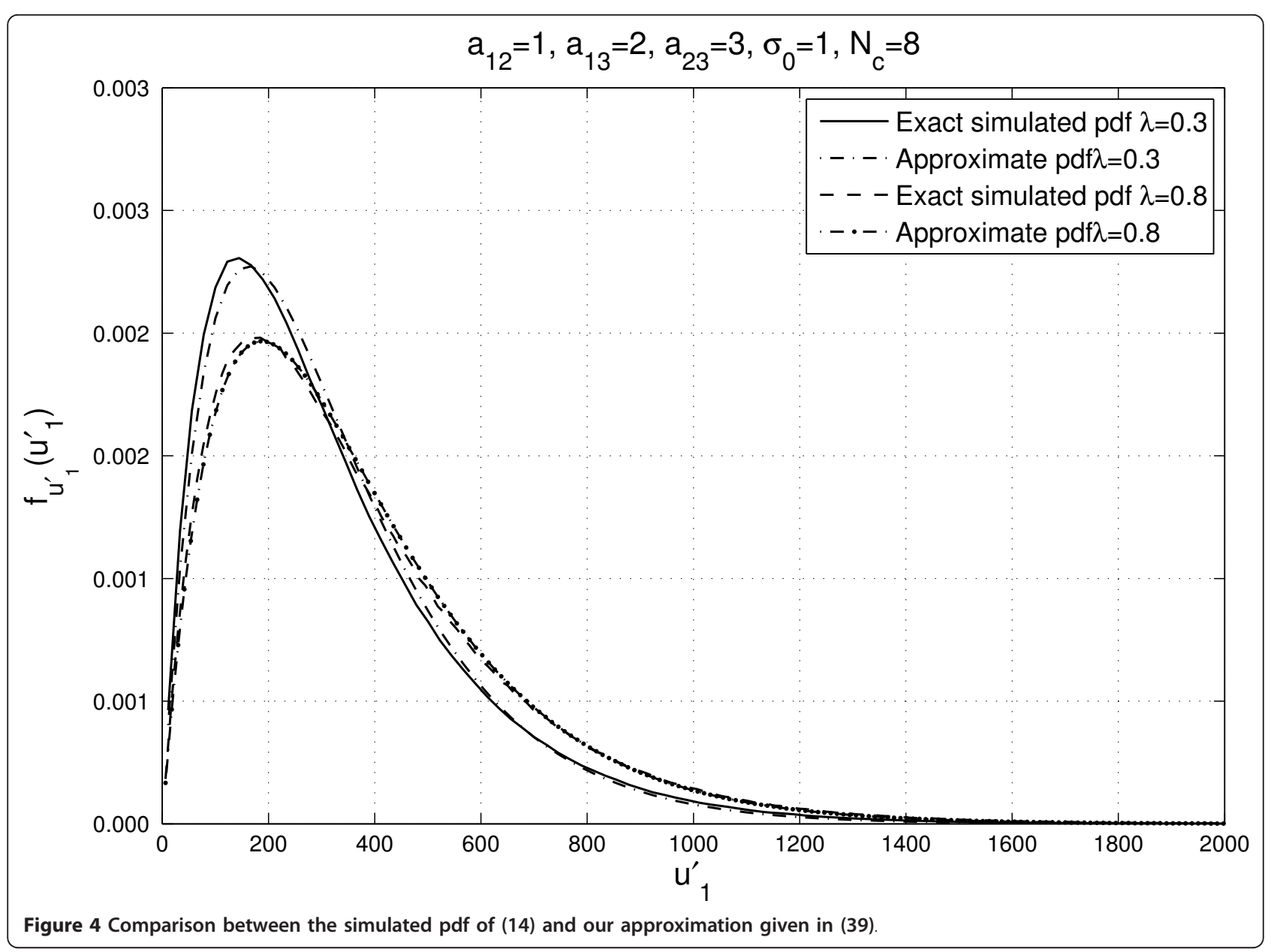

the cases, the approximation fits very well the exact PDF of $U_{2}$.

Using (39) and (42) into (17), it is possible to obtain a very accurate approximate bit error probability. Fortunately, both integrals can be found in a closed-form solution as

$$
\int_{0}^{\infty} Q\left(\sqrt{u_{1}}\right) \frac{4 u_{1}}{\gamma_{1}^{2}} e^{-2 u_{1} / \gamma_{1 d_{1}}}=\frac{1}{2}\left(1-\frac{\sqrt{\gamma_{1}}\left(\gamma_{1}+6\right)}{\left(\gamma_{1}+4\right)^{3 / 2}}\right)
$$

and

$$
\int_{-\infty}^{\infty} Q\left(u_{2}\right) \frac{1}{\sqrt{2 \pi v^{2}}} \mathrm{e}^{-\frac{\left(u_{2}-\gamma_{2}\right)^{2}}{2 v^{2}}} \mathrm{~d} u_{2}=Q\left(\frac{\gamma_{2}}{\sqrt{1+v^{2}}}\right)
$$

All these calculations lead to the approximate bit error probability for the $\lambda$-MRC detector as shown in (41), where $\gamma_{12}$ is given in (13), $\gamma_{1}$ is given in (40), $\gamma_{2}$ is given in (43), and $v_{2}$ is given in (44).

Assuming an equal power allocation scheme $\left(a_{12}=\right.$ $a_{13}=a_{23}=a$ ), Figure 6 shows the comparison between the theoretical bit error probability presented in (17) using the exact PDFs (28) and (31) and our approximation given in (41). We can observe that both curves are almost the same, validating our approximation.

Our results are quite exact for a different power allocation scheme as well. This can be seen in Figure 7, where a comparison between the exact simulated bit error probability and our approximation given in (41) was performed. In this figure, the following parameters were used $\alpha_{10}=\alpha_{20}=1$ and $\alpha_{12}=0.8$.

The final approximate expression allows us to determine the optimal value for $\lambda$ in each case. As stated in [1], when the BS believes that the inter-user channel is "perfect", then $\lambda=1$ and the optimal detector turns out to be the maximal ratio combining [7]. As the inter-user channel becomes more unreliable, i.e., as $P_{e_{12}}$ increases, the value of the best $\lambda$ decreases toward to zero. In order to demonstrate this behavior, Figure 8 shows the optimized $\lambda^{*}$ versus the inter-user channel parameter $\alpha_{12}$. This curve was obtained using computational optimization techniques that minimizes our approximate bit error probability (41) with respect to $\lambda$ for each value of the inter-user channel parameter, $\alpha_{12}$. The direct 


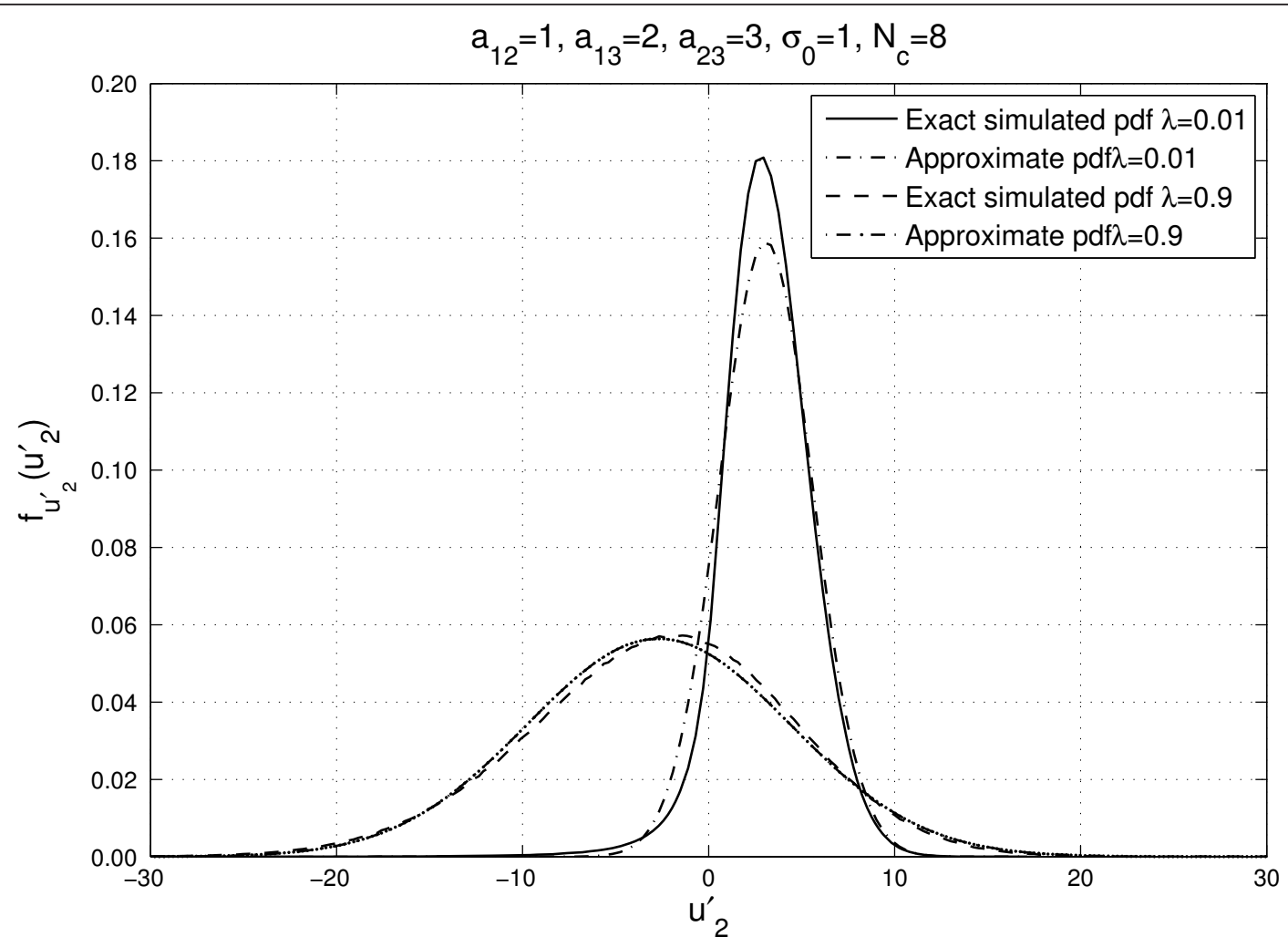

Figure 5 Comparison between the simulated PDF of (15) and our approximation given in (42)

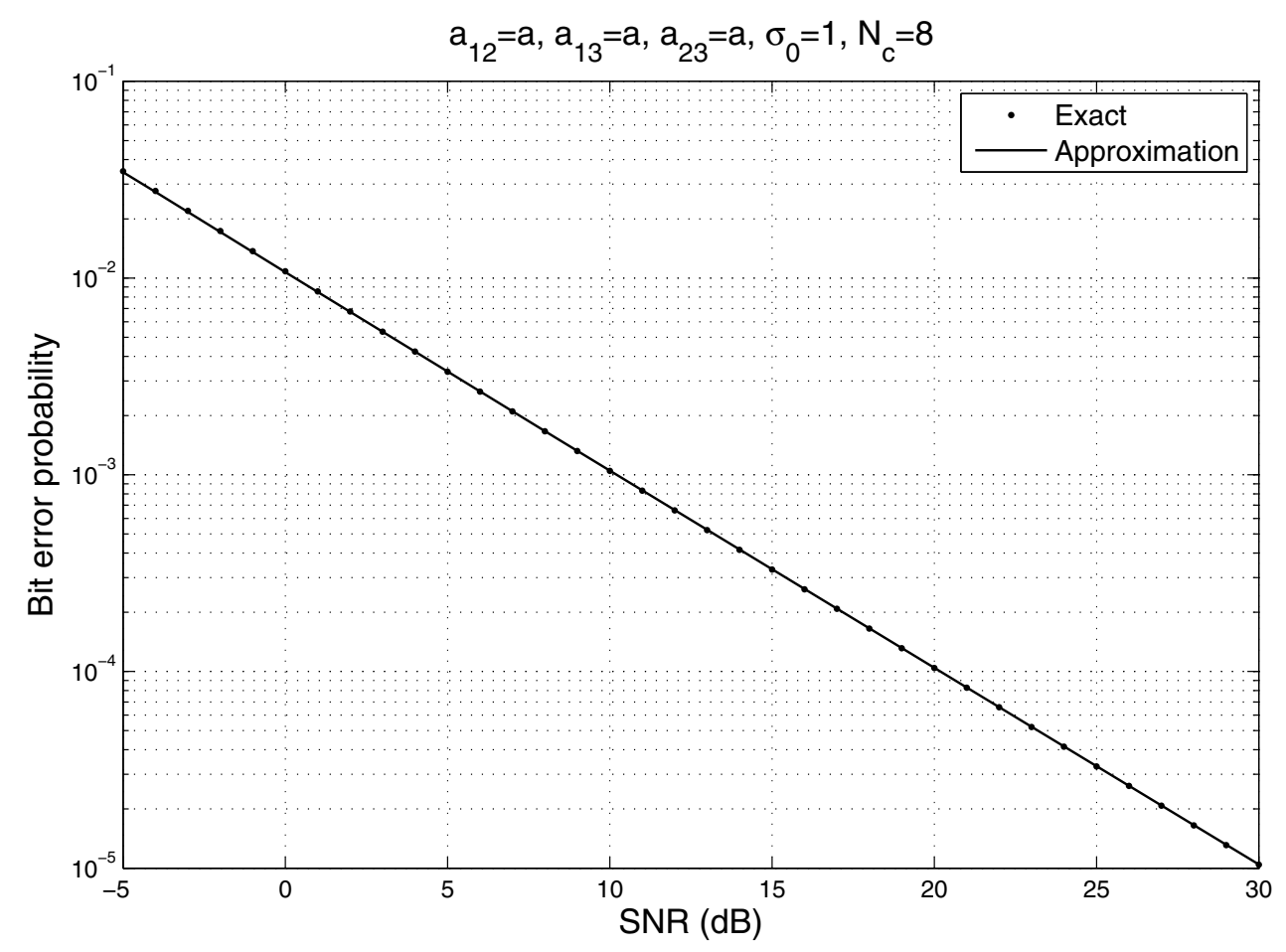

Figure 6 Comparison between exact and approximate bit error probability using an equal power allocation scheme with $\lambda=0.5$. 


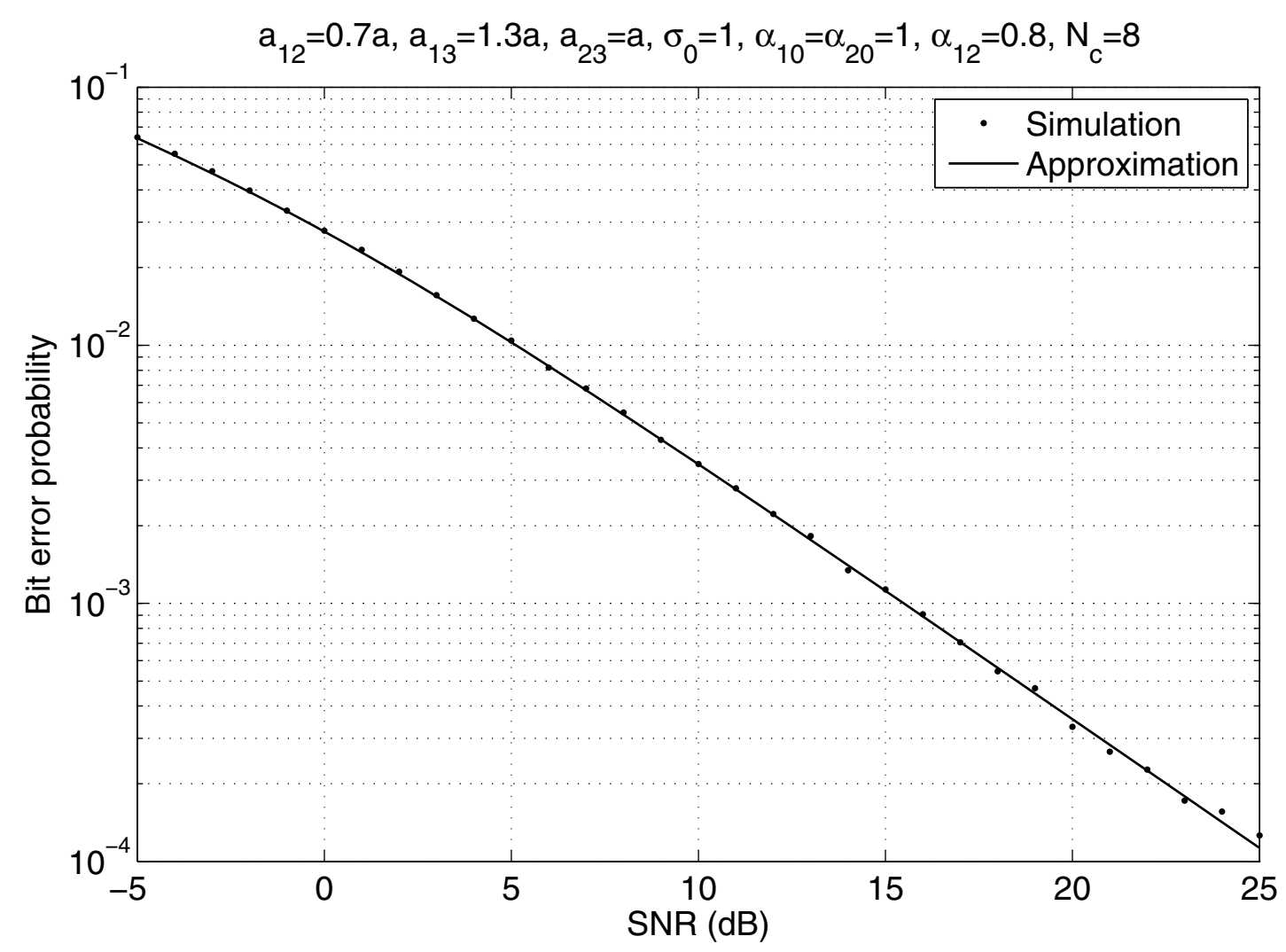

Figure 7 Comparison between exact and approximate bit error probability for a non equal power scheme allocation $\lambda=0.5$.

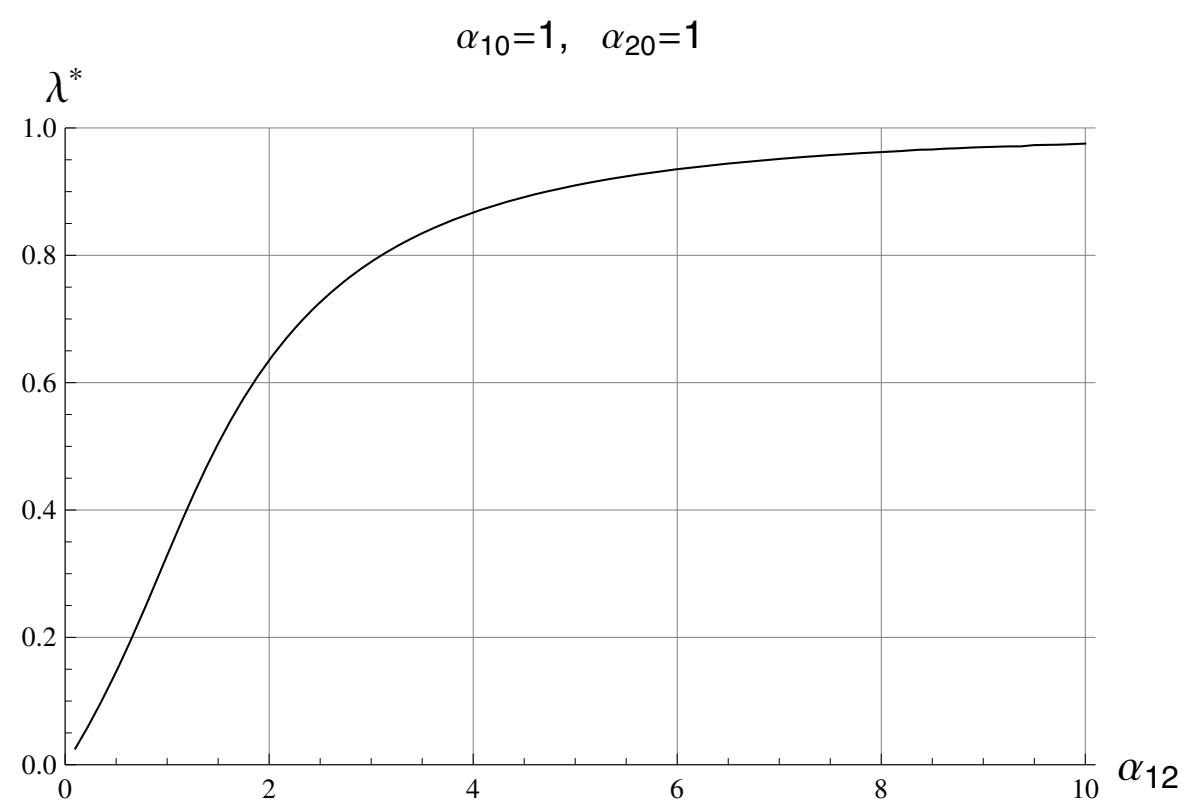

Figure 8 Optimized $\lambda^{*}$ versus $\alpha_{12}$. 
channel parameters $\alpha_{10}=\alpha_{20}=1$ were kept constant, and the equal power allocation scheme $\left(a_{12}=a_{13}=a_{23}\right.$ =1) was adopted.

\section{Conclusions}

In this paper, an exact and approximate expression for the average bit error probability under Rayleigh fading for the $\lambda$-MRC presented in [1] was obtained.

The exact expression was obtained under the condition of an equal power allocation scheme. The expression was validated through simulations showing a perfect agreement between exact and simulated curves.

In order to reduce the complexity of the exact expression, a very accurate approximation was presented as well. The approximate expression is valid for any values of $\lambda, a_{12}, a_{13}$, and $a_{23}$. The expression has been validated by simulation for a variety of values showing a small difference between the exact and approximate curves.

Both expression can be very important in many situations where the performance of a cooperative system employing CDMA should be evaluated.

\section{Competing Interests}

The authors declare that they have no competing interests.

\section{Appendix}

$$
\begin{gathered}
R_{1}=2 M_{1}+M_{2}+\frac{M_{3}}{M_{2}} \\
R_{2}=24 k_{20} \lambda^{2} \mu_{2}^{2} A_{2} \lambda_{1} \sqrt{\frac{3}{R_{1}}}+8 M_{1}-2 M_{2}-\frac{2 M_{3}}{M_{2}} \\
M_{1}=2 A_{2}^{2} \lambda \lambda_{1} k_{20}^{2}+\lambda_{2} u_{2}^{2} \\
M_{2}=\sqrt[3]{E+2\left(G+A_{2} k_{20} \lambda \lambda_{1} u_{2}^{2} \sqrt{27 F}\right)} \\
M_{3}=16 A_{2}^{4} \lambda^{2} \lambda_{1}^{2} k_{20}^{4}- \\
4 A_{2}^{2} \lambda\left(2 \lambda^{3}+5 \lambda^{2}+2 \lambda-1\right) u_{2}^{2} k_{20}^{2}+\lambda_{2}^{2} u_{2}^{4} \\
E=-\lambda_{2}^{3} u_{2}^{6}+6\left(A_{2} K_{20}\right)^{2} \lambda \lambda_{5} u_{2}^{4}- \\
24\left(A_{2} K_{20}\right)^{4} \lambda^{2} \lambda_{1}^{2} \lambda_{3} u_{2}^{2} \\
F=-16\left(A_{2} k_{20}\right)^{6} \lambda^{2} \lambda_{1}^{2} \lambda_{4}+8\left(A_{2} k_{20}\right)^{4} \lambda \lambda_{7} u_{2}^{2}- \\
\left(A_{2} k_{20}\right)^{2} \lambda_{6} u_{2}^{4}+\lambda_{2}^{3} u_{2}^{6} \\
G=32\left(A_{2} k_{20}\right)^{6} \lambda^{3} \lambda_{1}^{3} \\
\lambda_{1}=\lambda+1 \\
\lambda_{2}=\left(\lambda^{2}+1\right) \\
\lambda_{3}=\left(2 \lambda^{2}+3 \lambda-1\right) \\
\lambda \lambda_{4}=\left(5 \lambda^{2}+2 \lambda+1\right) \\
\lambda_{6}=\left(13 \lambda^{6}+28 \lambda^{5}-34 \lambda^{3}-12 \lambda^{2}-8 \lambda+1\right) \\
\lambda_{7}=\left(7 \lambda^{5}+22 \lambda^{4}+17 \lambda^{3}+3 \lambda^{2}-1\right) \\
\left(2 \lambda^{5}+5 \lambda^{4}-5 \lambda^{3}-14 \lambda^{2}-7 \lambda-1\right)
\end{gathered}
$$

Received: 11 February 2011 Accepted: 10 November 2011

Published: 10 November 2011

\section{References}

1. A Sendonaris, E Erkip, B Aazhang, User cooperation diversity Part II: Implementation aspects and perfomance analysis. IEEE Trans Commun. 51(11), 1939-1948 (2003). doi:10.1109/TCOMM.2003.819238

2. EC van der Meulen, Three-terminal communication channels. Adv Appl Probab. 3(1), 120-154 (1971). doi:10.2307/1426331

3. TM Cover, A El Gamal, Capacity theorem for the relay channel. IEEE Trans Inform Theory. 25(5), 572-584 (1979). doi:10.1109/TIT.1979.1056084

4. A Nosratinia, TE Hunter, A Hedayat, Cooperative communication in wireless networks. IEEE Commun Mag. 42(10), 74-80 (2004). doi:10.1109/ MCOM.2004.1341264

5. JN Laneman, DNC Tse, GW Wornell, Cooperative diversity in wireless networks: Efficient protocols and outage behavior. IEEE Trans Inform Theory. 50(12), 3062-3080 (2004). doi:10.1109/TIT.2004.838089

6. A Sendonaris, E Erkip, B Aazhang, User cooperation diversity Part I: System description. IEEE Trans Commun. 51(11), 1927-1938 (2003). doi:10.1109/ TCOMM.2003.818096

7. JG Proakis, Digital Communications, 4th ed. (McGraw-Hill, New York, 2001)

8. S A Papoulis, Unnikrishna Pillai, Probability, Random Variables and Stochastic Processes 2001, 4th ed. (McGraw-Hill, 2002)

doi:10.1186/1687-1499-2011-166

Cite this article as: Inga and Fraidenraich: Average bit error probability for the $\lambda$-MRC detector under Rayleigh fading. EURASIP Journal on Wireless Communications

and Networking 2011 2011:166.

\section{Submit your manuscript to a SpringerOpen ${ }^{\mathcal{O}}$ journal and benefit from:}

- Convenient online submission

- Rigorous peer review

- Immediate publication on acceptance

- Open access: articles freely available online

- High visibility within the field

- Retaining the copyright to your article

Submit your next manuscript at $\gg$ springeropen.com 